\title{
Analysis of Key Technologies of Digital Library Based on Cloud Computing
}

\author{
Guo Xin \\ School of Software Service Outsourcing \\ Jishou University \\ Zhangjiajie, China \\ jianghai079@126.com
}

\begin{abstract}
With the development of information technology, the library has changed greatly, and the target of the intensive literature information resources has been seriously challenged. The existing mode of information resources has gradually changed from the analog to digital state. The digital library began to enter the era of cloud computing. Cloud computing as a suitable for the application of the library architecture model, can be dispersed digital information resources integration, the intensification of the digital library, the construction and sharing of digital resources to provide a new solution. At first, this paper analyzes the concept of cloud computing and related technology, and then introduces the needs of the construction of digital library, finally to cloud computing in the construction of digital library applications. Secondly, it discusses how to design the architecture of Digital Library Based on cloud computing technology. From the logical structure of the digital library, the digital library cloud service platform, the implementation strategy of the digital library and the operation flow of the digital library. Finally, the design and implementation of a digital library is designed, which is composed of information resource management system, platform service system, information retrieval system, information system, information distribution system and information processing system. And gives the implementation process using Google and Amazon cloud services platform.
\end{abstract}

Keywords- Cloud computing; digital library; cloud service platform; virtualization technology; resource management

\section{INTRODUCTION}

Internet technology continues to move forward, people are increasingly accustomed to access through the Internet and access to information resources, the business scope of the traditional library is based on paper resources, which resulted in their crisis, but also unprecedented[1]. Digital library is a new medium, which can provide a huge amount of information media came into being. In the field of digital library, information with numerous forms: image, text, audio, video, and so on, in the digital library, this kind of multimedia information is indispensable[2]. So the digital library to manage the amount of data is growing, coupled with the network technology has gained unprecedented rapid development, coupled with the mobile broadband network into people's vision, mobile intelligent terminal has been widely spread, and the Internet is connected to the mobile device is also growing. On the Internet, its system load is also growing, which requires that it must have a large amount of data and the ability to digest and organize[3] data. In this case, the traditional digital library service construction mode has appeared to be inadequate, from the development of the digital library, the reader suffered from the first generation of the Internet to the second generation of the Internet between the transformation, at present, the third generation of Internet technology has quietly come to people's lives. In the same place, the traditional digital library also has a good opportunity, but also faces enormous challenges[4]. In Google, Amazon, Microsoft and other information technology leadership, the third generation of Internet technology, the symbol of the "cloud computing", has become a new network technology. Based on the distributed computing model, cloud computing, it can be more secure and effective. It can meet the needs of the construction of Digital Library in the future[5].

Cloud computing is developed by Computing Parallel, Grid Distributed (Computing), distributed processing (Computing) and so on[6]. It can also be said that the commercial development of such a concept of computer science is the cloud computing. From the nature of cloud computing, most of the computing tasks are distributed in the pool of resources by the computer, so that customers can be users according to their own needs to get related services, which includes the computing power, information services and storage space, etc.. Because the maturity of cloud computing is very high, it is a result of the evolution of multi class hybrid technology, and Amazon, YAHOO and Microsoft and other large companies to become the leader of cloud computing[7]. Accordingly, the scholars to cloud computing service model analogy model for power generation, which means computing resources and water, electricity, gas and other resources as provided by the service provider, does not require each user self-produced self, user quantity or pay it on time, computing power via the Internet transmission greatly reduce the development cost[8]. Cloud computing as a IT infrastructure and service delivery and service model, will profoundly affect the future of the operation and service mode, the traditional library and digital library in the future development, providing a full range of guidance and inspiration, but also for the traditional library provides a new operation mode, the library's cloud era is approaching.

In our country, the development of cloud computing is also very rapid. The library industry has gradually paid attention to the development of cloud computing technology and Library in this background. The academic committee of the Chinese Library Society held a symposium on "cloud computing and library" in Shanghai in September 2009, which means that the cloud computing service is truly in the library[9]. After this, the scholars and experts from the library, through discussing and analyzing, 
studied the following contents: cloud computing has brought about by the library, how to share information resources, cloud computing in the library and the difficulties in the use of cloud computing in the library. Since then, the research of cloud computing in the library has become a hot spot.

\section{TheOretiCAL OVER VIEW OF DigITAL LIBRARY BASED ON CLOUD COMPUTING}

Cloud computing to the existing computing resources and the use of a model to bring tremendous changes[10]. All services will also enable the concept of the digital library to benefit from the side. There is no doubt that cloud computing will have a profound impact on the development and construction of the digital library, and the application of cloud computing technology in the construction of digital library has a broad prospect. This chapter first analysis of cloud computing concept and the related technology, then introduces the demand of the construction of the digital library, finally to cloud computing in the construction of digital library applications.

$\mathrm{PaaS}$ is the trend of the development of SaaS technology, the main face of the user is the development of personnel. PaaS can bring higher performance, more personalized service to customers. Manufacturers to provide customers with the development of the environment, hardware resources, server platforms and other services, users on its platform to customize the development of their own applications and communicate to other customers through their Internet or servers. PaaS provides users with the development of the intermediate platform, and provides application development, testing, application server, database and application services. And SaaS products flourishing compared to PAAS products are mainly oriented to streamline, which compared to the well-known products have: force.com, Google App Engine, windows Azure Platform and heroku.

IaaS is a managed type of hardware infrastructure that serves. Provide to the customer is to put a number of manufacturers of servers to the cloud infrastructure as a measure of service. It is combined into a virtual resource pool, including the memory, I/O devices, computing power, storage, and other services for the entire industry to provide the required Virtualization Server and resource storage. Users pay for the use of hardware facilities provided by the manufacturer. Users can from suppliers to obtain what he needs computing or storage resources to the loading of the application, and only need to pay for the rent that part of the resource, and the management of the complicated is to IAAs providers to be responsible. The most representative IaaS products are: EC2 IBM, Blue Cloud Cisco, UCS Amazon and Joyent.

PaaS is a more abstract level of resources. It provides running environment to the user program. Fault tolerance mechanism and dynamic expansion of PaaS itself. The user application for node matching problem does not require too much consideration. But at the same time, the user must comply with the specific calculation of the title and use a specific program to use a specific program to use, the user's autonomy has done a downgrade.

\section{RESEARCH ON THE ARCHITECTURE OF DIGITAL LIBRARY BASED ON CLOUD COMPUTING}

\section{A. Logical architecture of Digital Library Based on Cloud Computing}

The logical structure of the design scheme of the digital library based on cloud computing is divided into four layers. The first layer is the service layer, provides the account supervision, portal supervision, deployment services and user supervision. The second layer is the management layer, the resource monitoring service and operation scheduling functions; the third layer is the virtualization layer, the hardware virtualization and data resource virtualization; the fourth layer is the data source layer, including physical server, Internet and storage settings. IaaS, PaaS, SaaS are three types of cloud computing operation mode, different cloud computing providers to provide different patterns, the current and no unified logic system, so as to provide a reference for the research and comprehensive solution to different factory program structure.

\section{B. Architecture model of digital library cloud service platform}

In the digital library, the application service layer is in the third layer of cloud computing service system architecture, which is the focus of all the digital library cloud service system. Take service layer to provide application software and service, that is, the need for the digital library. The digital library uses the application software service to carry out, according to the user needs, through the lease use way, the software or the application program provides to the union hall and the reader use. Provide digital library core services, these services are mainly including books, digital asset management, public information services, personalized information services, information search, search, unified resource search, the theme of the subscription to push services, the original delivery services, technology services, technology assessment services, enterprise competitive intelligence services, information services, information services and statistical analysis, etc.. The user authority billing interface module focuses on the support of the payment interface, which includes user supervision, authority demonstration, platform billing and platform payment interface support. Regulatory and business support system to achieve a system of regulatory and business support to ensure the normal and stable operation of the system.

\section{DESIGN AND IMPLEMENTATION OF DIGITAL LIBRARY BASED ON CLOUd COMPUTING}

The design of Digital Library Based on cloud computing should consider the advantages and disadvantages of the existing library data platform. Based on the existing technology, the latest mobile communication technology, network database technology, etc., according to the principle of cloud computing, design a new digital library and its embedded in the existing library database services platform, forming a fast, high reliability and integrated information service platform. In view of this, this chapter analyzes and designs a set of information resource management system, which is a set of user service system, information resource retrieval system, 
information processing system, information release and processing, and the design scheme is analyzed in detail.

Combined with the general design principle of the university library system, the design of this system is divided into two main parts of the library management system and the user's personal information resource management system. The hardware structure design of the two main parts should be fully considered in the design of the data center. In the scheme design, the software system design of the two parts should be equipped with the appropriate hardware equipment to support, while ensuring that the different software modules can be integrated with the physical server and virtual server technology in two ways to support.

\section{SYSTEM ARCHITECTURE}

The information management system of digital library is designed by the way of centralized deployment, the database platform is designed, and the centralized deployment mode of the database is completely different from the way of integration. Integrated deployment refers to a set of database, the database needs to use the database to support and complete each other. The database set the deployment of the pointer to the corresponding deployment of each business system independent database, but the physical device uses the partition and other virtualization technology to achieve physical location.

Two kinds of database deployment plan can achieve the goal of the database, but the first way is more thorough database concentration, is achieved through the database application level, and the second way is through physical device virtual technology to achieve the database location integration. System architecture diagram as shown below:

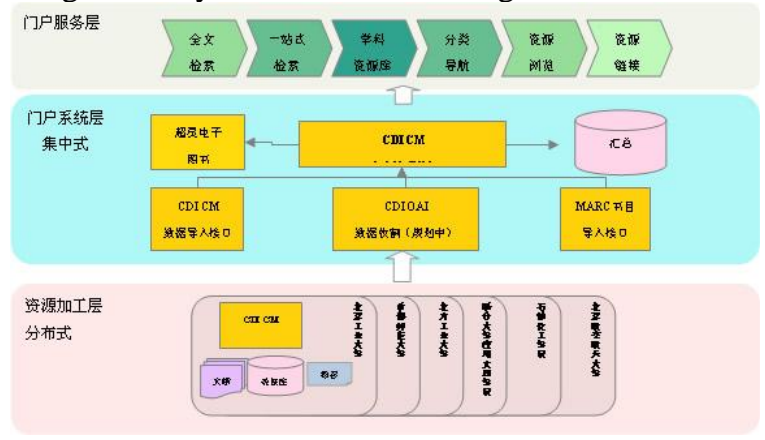

Figure 1. System framework

SNA technology, which can meet the needs of data center's information storage, is the current widespread application of storage technology. According to the resource shared memory of the storage area, it can realize the effective collection of the storage resource, and make it more flexible when the storage resource is allocated to the virtual machine. Using centralized storage method to carry on the virtual device virtualization can make the resource management of the storage platform, improve the practicability and reliability.

The computer server has multiple Ethernet interface card, which leads to the increase of network resource bandwidth, which makes the network practical efficiency higher. Most of the virtual machine services through the IP network to provide, the entire pool of resources through the IP network management. Due to the complexity of the digital library platform and the continuity of the current network, the existing network system platform and the current use of the switch, Gigabit network interface, etc. do not change and adjust, server virtualization use of existing basic resources. The database used in the system is shown in the picture below:

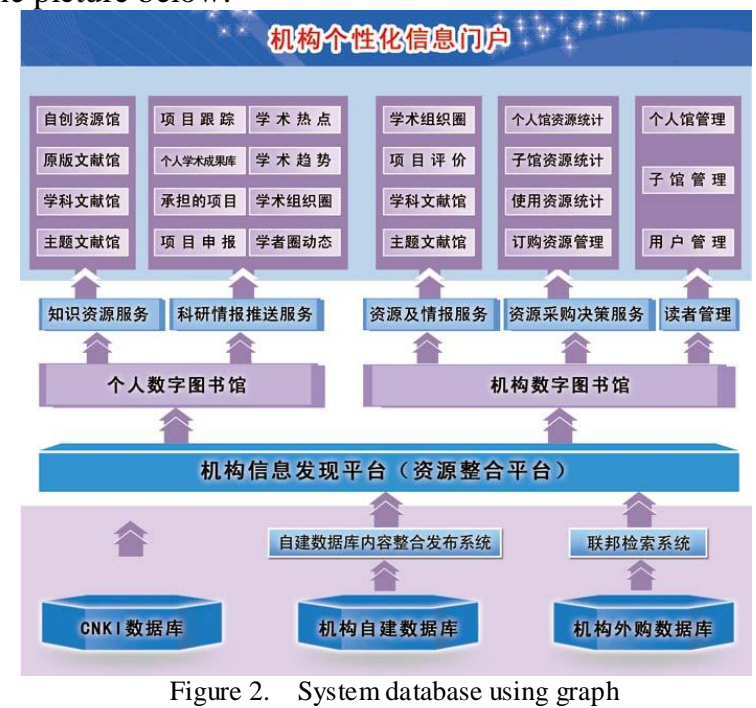

\section{CONCLUSIONS}

It is a long and arduous task to construct a successful digital library service system based on cloud computing. Need to the development of digital library practitioners and researchers in the third generation of the Internet era, to play their own advantages to the development of advanced information technology, continuous learning, continuous exploration, it is possible to adapt to the new situation of the development of digital libraries. Cloud computing has been concerned in the field of Library and information, cloud computing has become a kind of network computing model and business computing environment. The advantages of cloud computing technology, cloud computing digital library service model, the personal digital library based on cloud computing, and improve the efficiency of library network service, and create a new service model and service system, has become an urgent need to solve the problem. At present, China has not been a formal development plan of Digital Library Based on cloud computing. In recent years, we have tried to design a new digital library cloud service system, which is based on the research of the domestic and foreign literature data and analysis.

\section{ACKNOWLEDGMENT}

A Project Supported by Scientific Research Fund of Hunan Provincial Education Department(14B143) and Key laboratory for ecotourism of Hunan province of open fund for 2014.

\section{REFERENCES}

[1] Yong Xian Li, Xu Lun Luan, Sen Sen Li. Cloud computing technology in the library application $[\mathrm{J}]$, the Journal of the Library Science in Jiangxi, 2009 (1): 105-106.

[2] Yu L, Jiang T, Cao Y. Energy cost minimization for distributed internet data centers in smart microgrids considering power outages[J]. IEEE Trans. Parallel Distrib. Syst, 2015, 26(1):120-130. 
[3] Lu Xiaojuan, cloud computing and future library digital information resources construction $[\mathrm{J}]$, Sichuan Library Journal, 2009 (2): 23-24.

[4] Chen Kang, Zheng Weimin, Cloud Computing: system examples and research status [J], software journal, 2009, 20 (5): 1337-1348.

[5] Yang Mingfang, Yuan Xilin, cloud computing environment of digital library [J], library construction, 2009 (9): 7-12.

[6] Zhang Jian, cloud computing concepts and impact analysis of [J], telecommunications network technology, 2009 (1):335-339.

[7] Zhang D, Guo D, Chen F, et al. TL-plane-based multi-core energy-efficient real-time scheduling algorithm for sporadic tasks[J]. ACM Trans. Archit. Code Optim, 2012,8(4):47-48.
[8] Gu Zhimin, Zhang Jianxun, Zheng Chao, cloud computing research progress overview of $[\mathrm{J}]$, computer application research, No.2 Feb.2010, Vol.27.

[9] Breitgand D, Epstein A. Improving consolidation of virtual machines with risk-aware bandwidth oversubscription in compute clouds[C]. Proceedings of IEEE INFOCOM, 2012. 2861-2865.

[10] Zheng X, Cai Y. Energy-aware load dispatching in geographically located ineernet data centers $[\mathrm{J}]$. Sustainable Computing: Informatics and Systems, 2011, 1(4):275-285 